\title{
Developing and Evaluating Clinical Practice Guidelines: A Systematic Approach
}

Kathleen Phillips

\section{EDUCATIONAL OBJECTIVES}

After completion of this chapter, the reader should be able to:

1. Describe how to level the evidence used to develop and substantiate a practice protocol.

2. Differentiate among recommendations, guidelines, and practice protocols.

3. Evaluate clinical practice guidelines using Appraisal of Guidelines for Research and Evaluation (AGREE) II.

4. Identify the five steps of the process for discovery of best evidence and integration into practice.

5. Describe the best sources of evidence available to answer background/overview questions to support protocol development.

6. Describe the specialized evidence sources most appropriate to support protocol development for specific patients and/or problems.

\section{OVERVIEW}

Clinical decision-making that is grounded in the best available evidence is essential to promote patient safety and quality healthcare outcomes. With the knowledge base for geriatric nursing rapidly expanding, assessing geriatric clinical practice guidelines (CPGs) for their validity and incorporation of the best available evidence is critical to the safety and outcomes of care. In the second edition of this book, Lucas and Fulmer (2003) challenged geriatric nurses to take the lead in the assessment of CPGs, recognizing that, in the absence of best evidence, guidelines and protocols have little value for clinical decision-making. In the third edition of this book, Levin, Singleton, and Jacobs (2008) proposed a method for ensuring that the protocols included here were based on meta-analysis (if data are available), systematic review of the literature, and synthesis of best evidence.

The purpose of this chapter is to describe the process that was used to create the fourth, fifth, and the current sixth edition of Evidence-Based Geriatric Nursing Protocols for Best Practice. Before the third edition of this book, there was no standard process or specific criteria for protocol development, nor was there any indication of the "level of evidence" of each source cited in a chapter (i.e., the evidence base for the protocol). In the third and fourth editions of this book, the process previously used to develop the geriatric nursing protocols was enhanced and described in detail. That process differed from the procedures followed in the current edition. This chapter is a guide to 
understanding how the protocols contained in this book were developed and it details how to use a systematic, efficient, and evidence-based approach to discovering and evaluating evidence, which is the process needed to guide the assessment, development, and updating of practice protocols in any area of nursing practice.

\section{DEFINITION OF TERMS}

Evidence-based practice (EBP) is a framework for clinical decision-making that uses (a) the best available evidence, (b) the clinician's expertise, and (c) a patient's values and circumstances to guide judgments about a patient's personal health condition (Keefer \& Levin, 2013; Melnyk \& Fineout-Overholt, 2015; Straus, Glasziou, Richardson, $\&$ Haynes, 2010). Healthcare professionals often use the terms recommendations, guidelines, and protocols interchangeably, but they are not synonymous.

A recommendation is a suggestion for practice, not necessarily sanctioned by a formal, expert group. A CPG is an "official recommendation" or suggested approach to diagnose and manage a broad health condition or problem (e.g., heart failure, smoking cessation, or pain management). A protocol is a more detailed guide for approaching a clinical problem or health condition and is tailored to a specific practice situation. For example, guidelines for falls prevention recommend developing a protocol for toileting older, sedated, or confused patients (Gray-Miceli \& Quigley, 2013). The specific practices or protocols that each healthcare organization implements, however, are agency specific. The validity of any of these practice guides can vary depending on the type and the level of evidence on which they are based. Using standard criteria to develop or refine CPGs or protocols assures reliability of their content. Standardization gives both nurses, who use the guideline/protocol, and patients, who receive care based on the guideline/protocol, assurance that the geriatric content and practice recommendations are based on the best evidence.

In contrast to these practice guides, "standards of practice" are not specific or necessarily evidence based; rather, they are a generally accepted, formal, and published framework for practice. As an example, the American Nurses Association document, Nursing: Scope and Standards of Practice, Third Edition, contains a standard regarding nurses' accountability for making an assessment of a patient's health status (American Nurses Association, 2015). The standard is a general statement, whereas a protocol may specify the measurement tool(s) to use in that assessment-for example, STRATIFY, an instrument used to measure the risk of falls (Smith, Forster, \& Young, 2006).

\section{The AGREE (Appraisal of Guidelines for Research and Evaluation) and AGREE II Instruments}

The AGREE instrument (www.agreetrust.org/resource -centre/the-original-agree-instrument), originally created and evaluated by a team of international guideline developers and researchers for use by the National Health Service, has been revised and updated and remains a generic tool designed primarily to help guideline developers and users assess the methodological quality of guidelines (Brouwers et al., 2010). This appraisal includes evaluation of the methods used to develop the CPG, assessment of the validity of the recommendations made in the guideline, and consideration of factors related to the use of the CPG in practice. Although the AGREE instrument was created to critically appraise CPGs, the process and criteria can also be applied to the development of clinical practice protocols. Thus, the AGREE instrument has been expanded for that purpose to standardize the creation and revision of the geriatric nursing practice protocols in this book.

The initial AGREE instrument and the one used for clinical guideline/protocol development in the third edition of this book has six quality domains: scope and purpose, stakeholder involvement, rigor of development, clarity and presentation, application, and editorial independence. A total of 23 items divided among the domains were rated on a 4-point Likert-type scale from "strongly disagree" to "strongly agree." Appraisers evaluate how well the guideline they are assessing meets the criteria (i.e., items) of the six quality domains. For example, when evaluating the rigor of development, appraisers rated seven items. The reliability of the AGREE instrument is increased when each guideline is appraised by more than one appraiser. Each of the six domains receives an individual domain score and, based on these scores, the appraiser subjectively assesses the overall quality of a guideline.

Important to note, however, is that the original AGREE instrument was revised in 2009 (AGREE Next Steps Consortium, 2013) and is now called AGREE II, which is the version that we used for the fourth, fifth, and sixth editions of this book. The revision added one new item to the rigor of development domain. This is the current item 9, which underscores the importance of evaluating the evidence that is applied to practice. Item 9 reads: "The strengths and limitations of the body of evidence are clearly described" (Exhibit 1.1). The remainder of the changes included a revision of the Likert-type scale used to evaluate each item in the AGREE II, a reordering of the number assigned to each item based on the addition of the new item 9, and minor editing of items for clarity. No other substantive changes were made. Exhibit 1.1 includes the items that are in the 


\section{EXHIBIT 1.1}

\section{Sample Domain and Items From the AGREE II Instrument for Critical Appraisal of Clinical Practice Guidelines}

\footnotetext{
Domain 3: Rigor of Development

7. Systematic methods were used to search for evidence.

8. The criteria for selecting the evidence are clearly described.

9. The strengths and limitations of the body of evidence are clearly described.

10. The methods for formulating the recommendations are clearly described.

11. The health benefits, side effects, and risks have been considered in formulating the recommendations.

12. There is an explicit link between the recommendations and the supporting evidence.

13. The guideline has been externally reviewed by experts prior to its publication.

14. A procedure for updating the guideline is provided.
}

AGREE, Appraisal of Guidelines for Research and Evaluation. Source: Reprinted from AGREE Next Steps Consortium. (2013). AGREE I/ instrument. Retrieved from http://www.agreetrust.org/ wp-content/uploads/2013/10/AGREE-II-Users-Manual-and -23-item-Instrument_2009_UPDATE_2013.pdf, by permission of Melissa Brouwers

rigor of development domain and were used for evaluation of evidence in the current edition of this book. A 2013 update of the AGREE II instrument (www.agreetrust.org/resourcecentre/agree-ii-training-tools) includes a history of the project, information about language translations, and enhanced online training tools freely available to support guideline developers (AGREE Enterprise, 2014).

The rigor of development section of the AGREE instrument provides standards for literature searching and documenting the databases and terms searched. Adhering to these criteria to find and use the best available evidence on a clinical question is critical to the validity of geriatric nursing protocols and ultimately to patient safety and outcomes of care.

Published guidelines can be appraised using the AGREE II instrument. In the process of guideline development, however, the clinician is faced with the added responsibility of appraising all available evidence for its quality and relevance. In other words, how well does the available evidence support recommended clinical practices? The clinician needs to be able to support or defend the inclusion of each recommendation in the protocol based on its level and quality of evidence. To do so, the guideline must reflect a systematic, structured approach to find and assess the available evidence.

\section{Searching for the Best Evidence}

Models of EBP describe the evidence-based process in five steps (Melnyk \& Fineout-Overholt, 2015; Titler, 2010):

1. Develop an answerable question.

2. Locate the best evidence.

3. Critically appraise the evidence.

4. Integrate the evidence into practice using clinical expertise with attention to patient's values and perspectives.

5. Evaluate the outcome(s).

Although the evidence-based process encompasses these five steps, for the purposes of this volume of protocols and their development, this chapter focuses on the first three steps in more detail.

\section{Step 1: Develop an Answerable Question}

Developing an answerable question is critical before one can choose relevant sources to search. The information needed may be in the form of a specific "foreground" question (one that is focused on a particular clinical issue) or it may be a broad question (one that asks for overview information about a disease, condition, or aspect of healthcare; Melnyk \& Fineout-Overholt, 2015; Straus et al., 2010) to gain some background of the practice problem and interventions, and gain insight into its significance. Background information includes both internal data from a specific agency and external data to place the health condition or problem in a broader societal context. Internal data usually include quality metrics from the healthcare agency in conjunction with healthcare providers' observations. External data might require a search for local and/ or national benchmarking data and prevalence statistics as well as general literature describing the local problem as one that goes beyond a specific healthcare setting, population, or intervention.

An example of a background query might be one that seeks data: What is the prevalence of falls in older residents in a long-term care facility? Should these data demonstrate an unacceptable fall rate compared with national benchmark and safety target statistics, then the local problem can be shown to have significance beyond the specific clinical agency. A broad research query (an example of an overarching background question) related to a larger category of disease or health problem and encompassing multiple interventions 
might be: What is the best evidence for fall prevention in hospitalized older adults? The first place to search for evidence would be the ECRI Institute's Guidelines Trust ${ }^{\mathrm{TM}}$ (http:// guidelines.ecri.org), as described in Table 1.1.

A related question-What is the best evidence for falls prevention for the elderly in hospitals and long-term care facilities? - is addressed in a systematic review (Cameron et al., 2012) from the Cochrane Library, as cited in Table 1.1. The Cochrane Library of Systematic Reviews contains rigorous and comprehensive narrative and statistical (meta-analyses) reviews that synthesize multiple studies of interventions. The information contained in this review synthesizes multifactorial interventions and may help to further focus the inquiry into a question about the effectiveness of a specific intervention.

A similar example (Slade, 2013) in Table 1.1 cites a Joanna Briggs Institute's evidence summary, which answers a general background or overview question: What is the evidence regarding specific interventions to prevent falls in older adults?

Once the overall evidence regarding a background question is uncovered, the question can be narrowed into a specific "PICO" format to specify the intervention or assessment tool being examined (Straus et al., 2010, p. 15). PICO stands for:
1 $\mathrm{P}=$ Population or patient problem
- I $=$ Intervention
- $\mathrm{C}=$ Comparison group or standard practice
O $=$ Outcomes
$\mathrm{T}=$ Time

The focused clinical or PICO question now specifies a patient problem or population and focuses on a specific intervention-for example, Does the introduction of a video-based educational module for staff decrease the rate of falls for hospitalized patients?

A case study (Cangany et al., 2015), located in the Cumulative Index to Nursing and Allied Health Literature (CINAHL) article database, provides an example of evidence in a specialized hospital setting. Foreground questions are best answered by individual primary studies or syntheses of multiple studies, such as systematic reviews or meta-analyses. PICO templates work best to gather the evidence for focused clinical questions. In the question mentioned earlier, the problem was identified as a hospital progressive care unit with a fall rate higher than the $\mathrm{Na}$ tional Database of Nursing Quality Indicators (NDNQI) benchmark, for a population of patients with heart disease. The intervention was the implementation of an educational video for staff, along with improved signage, improved documentation of bed alarm usage, a fall risk assessment, and a "patient/family fall teaching contract." The comparison implied was the usual care, and the outcome measures were both a reduction in the fall rate and the costs associated with the intervention versus the change in fall rate.

\section{Step 2: Locate the Best Evidence}

Step 2, locate the evidence, requires an evidence search based on the elements identified in the clinical question. Gathering the evidence for the protocols in this book presented the challenge of conducting literature reviews encompassing both the breadth of overview information as well as the depth of specificity represented in high-level systematic reviews and clinical trials to answer specific clinical questions.

Not every nurse, whether he or she is a staff nurse, advanced practice nurse, educator, or administrator, has developed proficient database search skills to conduct a literature review to locate evidence. Beyond a basic knowledge of Boolean logic, truncation, and applying categorical limits to filter results, competency in "information literacy" requires experience with the idiosyncrasies of databases, selection of terms, and ease with controlled vocabularies and database functionality (Association of College \& Research Libraries, 2013). Many nurses report that limited access to resources, gaps in information literacy skills, and, most of all, a lack of time are barriers to "readiness" for EBP (Pravikoff, Tanner, \& Pierce, 2005).

The digital age presents both consumers of research evidence and researchers with an array of tools for searching, managing, and citing both the published literature and the unpublished literature. The ever-changing electronic environment provides an array of search engines, "apps," and specialized discovery tools. Such an environment can be daunting and often overwhelming to novice and experienced users alike. Research portals promoting "one-box" search tools purport comprehensiveness, yet search results are often vast and unfiltered. The apparent ease of keyword searching invites cherry picking from the first few pages of results and can unwittingly introduce "search bias" (Wentz, 2002) into the quest for evidence, thus negating the sophisticated methodologies that were employed in primary searches to decrease experimenter bias and increase quality of evidence.

Health sciences librarians as intermediaries have been called "an essential part of the health care team by allowing knowledge consumers to focus on the wise interpretation and use of knowledge for critical decision making, rather than spending unproductive time on its access and retrieval" (Homan, 2010, p. 51). The Cochrane Handbook points out the complexity of conducting a systematic literature review 
TABLE 1.1

Selected Databases, Examples of Types of Questions, Sample Citations, and Level of Evidence of Citation

\begin{tabular}{l} 
Database/Description/Access \\
\hline PubMed/MEDLINE \\
Premier biomedical database produced \\
by the U.S. National Library of Medicine \\
containing more than 25 million citations \\
for biomedical literature from MEDLINE, life \\
science journals, and online books. \\
http://pubmed.gov
\end{tabular}

\section{CINAHL}

Cumulative Index to Nursing and Allied Health Literature (authoritative index for more than 5,000 nursing and allied health journals). http://health.ebsco.com/products/ the-cinahl-database/allied-health-nursing

\section{PsycINFO}

Indexes the professional and academic literature in the behavioral sciences and mental health, including medicine, psychiatry, nursing, sociology, pharmacology, physiology, and linguistics.

http://www.apa.org/pubs/databases/psycinfo

\begin{abstract}
Joanna Briggs Institute EBP Database
Evidence summaries (short abstracts that summarize existing international evidence on common healthcare interventions and activities based on structured searches of the literature and selected evidence-based healthcare databases).

http://connect.jbiconnectplus.org
\end{abstract}

Cochrane Database of Systematic Reviews Produced by the Cochrane Library, one of the six databases that contain different types of high-quality, independent evidence to inform healthcare decision-making.

http://www.cochranelibrary.com

\section{ClinicalTrials.gov}

A service of the U.S. National Institutes of Health, an international registry of publicly and privately supported clinical studies of human participants.

https://clinicaltrials.gov

\section{ECRI Institute Guidelines Trust ${ }^{\mathrm{TM}}$}

A public resource for evidence-based clinical practice guidelines.

http://guidelines.ecri.org

$\begin{array}{ll}\text { Overview Question } & \begin{array}{l}\text { PICO or Focused } \\ \text { Clinical Question }\end{array} \\ & \text { In hospitalized elders, } \\ \text { does the STRATIFY falls } \\ \text { risk assessment tool } \\ \text { predict falls in hospital } \\ \text { and after discharge? }\end{array}$

Does the introduction of an educational video recording for staff decrease the rate of falls for hospitalized patients?

Does exercise improve static and dynamic balance and dual-task ability in healthy older adults?

What is the evidence regarding specific interventions to prevent falls in older adults?

What is the best evidence for falls prevention for the older adult in hospitals and long-term care facilities?

What is the best evidence for fall prevention in hospitalized older adults?

Cangany, Back, Level V Hamilton-Kelly, Altman, and Lacey (2015)

Gobbo, Bergamin, Sieverdes, Ermolao, and Zaccaria (2014)

Slade (2013) Level VI

Cameron et al. Level I (2012)

Are high-intensity exercise programs an effective intervention for patients with Parkinson's disease, compared with the usual care (lowintensity group therapy)? Guidelines Trust (ECRI, 2019)
ECRI Institute

ClinicalTrials.gov NA (2014)

Level I

EBP, evidence-based practice; PICO, population, intervention, comparison group or standard practice, outcomes.

Note: Not every question will have the Time component.

Source: ECRI Institute. (2019). Retrieved from https://guidelines.ecri.org/ 
and highly recommends enlisting the help of a healthcare librarian when searching for evidence (Lefebvre, Manheimer, \& Glanville, 2011).

\section{Search Strategies}

General or overview/background questions may be answered in textbooks, review articles, and "point of care" tools that aggregate overviews of best evidence, for example, clinical reference tools, systematic reviews, and synthesis tools. Locating systematic or narrative review articles or clinical guidelines based on systematic reviews may be helpful in the initial steps of gathering external evidence to support the significance of a problem before developing a narrower PICO question and investing a great deal of time in a question for which there might be limited evidence.

A search for individual studies in the published literature begins with database selection and translation of search terms into the controlled vocabulary of the database, if possible. In addition to the published literature, unpublished "grey" literature should also be considered. Grey literature, defined during the Fourth International Conference on Grey Literature, is the information "which is produced on all levels of government, academics, business and industry in print and electronic formats, but which is not controlled by commercial publishers" (National Information Center on Health Services Research and Health Care Technology, n.d., para 2). It includes resources such as theses, dissertations, conference proceedings, standards, technical documentation, newsletters, corporate organizations, and government documents. Although grey literature may not be peer-reviewed or evaluated, it is nonetheless a part of a comprehensive gathering of evidence as a source for CPGs and protocols. One example in Table 1.1, clinicaltrials.gov, lists a study that proposes an exercise program for patients who have Parkinson's disease, with the status "recruiting participants" as of 2014 (ClinicalTrials.gov, 2014). Trial registries and open-access repositories of clinical trials provide study criteria, outcome measures, and historical revisions to studies. They may be specialized for a particular kind of publication; for example, the Cochrane Library (www .cochranelibrary.com) and the PROSPERO database (www .crd.york.ac.uk/prospero), are both examples of systematic review protocol repositories. The major article databases for finding the best primary evidence for most clinical nursing questions are the CINAHL database (https://www.ebscohost .com/nursing/products/cinahl-databases), the MEDLINE, and the U.S. National Library of Medicine's premier biomedical article database (www.ncbi.nlm.nih.gov/pubmed). The PubMed interface to MEDLINE includes newly added citations to provide access to the most recently published literature. Another of the many sources of ongoing trials for nursing research is the Virginia Henderson Global Nursing e-Repository (www.sigmarepository.org). The Cochrane Library (which includes the Database of Systematic Reviews) and the Joanna Briggs publications (a paid subscription service including evidence summaries, practice sheets, and systematic reviews; http://connect.jbiconnectplus.org) are examples of synthesized, appraised sources of evidence for broad topic areas.

The AGREE II instrument was used as a standard against which we could evaluate the process for evidence searching and use in chapter and protocol development (AGREE Next Steps Consortium, 2013). Domain 3, rigor of development, Item 7, states: "Systematic methods were used to search for evidence" (AGREE Next Steps Consortium, 2013, p. 4). And the user's manual directs: "The search strategy should be as comprehensive as possible and executed in a manner free from potential biases and sufficiently detailed to be replicated" (p. 23). Taking a tip from the Cochrane Handbook, a literature search should capture both the subject terms and the methodological aspects of studies when gathering relevant records (Higgins \& Green, 2011). The following guidelines reflect the process used to gather evidence for this book's protocols and are recommended guidelines for conducting a literature search.

To facilitate replication and update of searches, save a search strategy listing the keywords/descriptors and search string used in each database searched (e.g., MEDLINE, PsycINFO, CINAHL, trial registries).

Specify the time period searched (e.g., 2014-2019).

- Specify the categorical limits or methodological filters used (e.g., the article type: "meta-analysis" or the "systematic review subset" in PubMed; the "methodology" limit in PsycINFO for meta-analysis or clinical trial; the "research" limit in CINAHL).

Aggregate and organize evidence in a bibliographic management tool (e.g., Endnote [www.endnote.com], Mendeley [www.mendeley.com], RefWorks [https://www .refworks.com/refworks2], or Zotero [www.zotero.org]). Gathering evidence to support broader topics, such as the protocols in this book, presents the searcher with a greater challenge. Limiting searches by methodology can unwittingly eliminate the best evidence for study designs that do not lend themselves to these methods. For example, a cross-sectional retrospective design may provide the highest level of evidence for a study that examines "nurses' perception" of the practice environment (Boltz et al., 2008).

A challenge to a searcher is the need to balance the comprehensiveness of recall (or "sensitivity") with precision ("specificity") to retrieve a manageable number of references. The Cochrane Handbook states: "Searches should seek high 
sensitivity, which may result in relatively low precision" (Lefebvre et al., 2011, Section 6.1). Thus, retrieving a large set of articles may include many irrelevant hits. Conversely, putting too many restrictions on a database search may exclude relevant studies. The goal of retrieving the relevant studies for broad topic areas requires "sacrificing precision" and manually filtering false or irrelevant hits. Pitfalls of computerized retrieval are justification for the review by the searcher to hand weed false hits from the retrieved list of articles.

The iterative nature of any literature search means that an initial set of relevant references for both broad or specific questions serves to point protocol authors toward best evidence as an adjunct to their own knowledge and their own pursuit of "chains of citation" (McLellan, 2001), related records, and their clinical expertise. For example, a core list of references on the topic of physical restraints might lead to exploring citations related to wandering, psychogeriatric care, or elder abuse.

\section{Step 3: Critically Appraise the Evidence}

Step 3, critically appraise the evidence, begins with identifying the methodology used in a study (often evident from reviewing the article abstract) followed by a critical reading and evaluation of the research methodology and results. The coding scheme described subsequently provides the first step in filtering retrieved studies based on research methods.

Levels of evidence (LOE) offer a schema that, once known, helps the reader to follow an efficient path for evidence searching as well as understand the relative value of the information presented to the clinical topic or question under review. There are many extant schemas used to identify the LOE of sources. Although multiple schemas exist, there are commonalities in their hierarchical structure, often represented by a pyramid or "publishing wedge" (DiCenso, Bayley, \& Haynes, 2009). The highest level of evidence is seen at the top of a pyramid, usually systematic reviews (meta-analyses) and CPGs based on high-level evidence. The LOE for the current edition can be seen in Figure 1.1 (Levin, 2011). A major difference exists between the former and current pyramids that are recommended for guiding your evidence search. Quantitative and qualitative research ask very different questions and thus cannot be included in the same hierarchy-of-evidence scheme. A hierarchy of qualitative evidence, developed by Daly et al. (2007), further aids researchers in the process of guiding the evidence search.

A Level I evidence rating is given to evidence from synthesized sources: systematic reviews, which can either be meta-analyses or structured integrative reviews of evidence, and CPGs based on Level I evidence as well as

\section{FIGURE 1.1}

\section{Level of evidence hierarchy.}

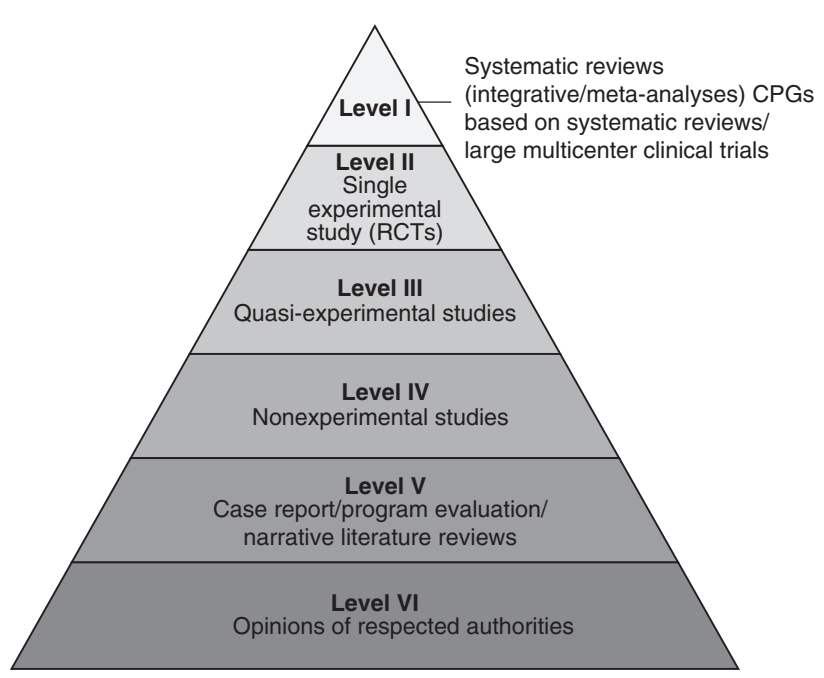

CPG, clinical practice guidelines; RCT, randomized controlled trial.

Source: Originally published in Levin, R. F. (2011). Edifices of evidence: The proliferation of pyramids. Research and Theory for Nursing Practice, 25(1), 8-10. doi:10.1891/1541-6577.25.1.8.

Reprinted with permission.

multicenter clinical trials. Evidence that is judged to be at Level II comes from a single randomized controlled trial. A quasi-experimental study, such as a nonrandomized controlled single group pretest/posttest, time series, or matched case-controlled study, is considered Level III evidence. Level IV evidence comes from a nonexperimental study, such as correlational descriptive research or case-control studies. A narrative literature review, a case report systematically obtained and of verifiable quality, or program evaluation data are rated as Level V. Level VI evidence is identified as the opinion of respected authorities (e.g., internationally or nationally known) based on their clinical experience or the opinions of an expert committee, including their interpretation of nonresearch-based information. This level also includes regulatory or legal opinions. Level I evidence is considered the strongest.

The critical appraisal of extant CPGs and research studies uses specialized tools designed to evaluate the quality of each type of evidence. Examples are the AGREE II instrument (which this volume of protocols conforms to), the Critical Appraisal Skills Programme (www.casp-uk .net), and the PRISMA checklist (a 27-item list of criteria for evaluation; www.prisma-statement.org) for reporting systematic reviews and meta-analyses, among others. 
This book provides the inclusion of the level and type of evidence for references in chapter citations, which leads to a recommendation for practice. Using this type of standard approach ensures that this book contains protocols and recommendations for use with geriatric patients and their families that are based on the best available evidence and a similar standard of evaluation.

\section{SUMMARY}

The protocols contained in this edition, therefore, have been refined, revised, and developed by the authors using the best available research evidence as a foundation, with the ultimate goal of improving patient safety and outcomes. The systematic process used for finding, retrieving, and disseminating the best evidence for this edition of Evidence-Based Geriatric Nursing Protocols for Best Practice provides a model for the use of research evidence in nursing education and in clinical practice. Translating nursing research into practice requires competency in information literacy, knowledge of the evidence-based process, and the ability to discern the context of a research study as ranked hierarchically. The following chapters and protocols present both overview (background) and foreground information in readiness for taking the next steps in the EBP process: Step 4: Integrate the evidence into practice using clinical expertise with attention to patient's values and perspectives, and Step 5: Evaluate the outcome(s).

\section{ACKNOWLEDGEMENTS}

The author expresses appreciation for the work of previous authors of this chapter: Rona F. Levin and Susan Kaplan Jacobs.

\section{REFERENCES}

AGREE Next Steps Consortium. (2013). AGREE II instrument. Retrieved from http://www.agreetrust.org/wp-content/ uploads/2013/10/AGREE-II-Users-Manual-and-23-item -Instrument_2009_UPDATE_2013.pdf

American Nurses Association. (2015). Nursing: Scope and standards of practice (3rd ed.). Silver Spring, MD: Author.

Association of College \& Research Libraries. (2013). Information literacy competency standards for nursing. Retrieved from http:// www.ala.org/acrl/standards/nursing

Boltz, M., Capezuti, E., Bowar-Ferres, S., Norman, R., Secic, M., Kim, H., ... Fulmer, T. (2008). Hospital nurses' perception of the geriatric nurse practice environment. Journal of Nursing Scholarship, 40(3), 282-289. doi:10.1111/ j.1547-5069.2008.00239.x
Brouwers, M. C., Kho, M. E., Browman, G. P., Burgers, J. S., Cluzeau, F., Feder, G., ... AGREE Next Steps Consortium. (2010). AGREE II: Advancing guideline development, reporting, and evaluation in health care. Preventive Medicine, 51(5), 421-424. doi:10.1016/j.ypmed.2010.08.005

Cameron, I. D., Gillespie, L. D., Robertson, M. C., Murray, G. R., Hill, K. D., Cumming, R. G., \& Kerse, N. (2012). Interventions for preventing falls in older people in care facilities and hospitals. Cochrane Database of Systematic Reviews, 2012(12), CD005465. doi:10.1002/14651858.CD005465.pub3

Cangany, M., Back, D., Hamilton-Kelly, T., Altman, M., \& Lacey, S. (2015). Bedside nurses leading the way for falls prevention: An evidence-based approach. Critical Care Nurse, 35(2), 82-84. Retrieved from http://ccn.aacnjournals.org/content/35/2/82.full

ClinicalTrials.gov. (2014). High-intensity exercise and fall prevention boot camp for Parkinson's disease. Retrieved from https:// clinicaltrials.gov/ct2/show/study/NCT02230267?term=falls + exercise\&rank=3

Daly, J., Willis, K., Small, R., Green, J., Welch, N., Kealy, M., \& Hughes, E. (2007). A hierarchy of evidence for assessing quality health research. Journal of Clinical Epidemiology, 60(1), 43-49. doi:10.1016/j.jclinepi.2006.03.014

DiCenso, A., Bayley, L., \& Haynes, R. B. (2009). Accessing pre-appraised evidence: Fine-tuning the $5 \mathrm{~S}$ model into a 6S model. Evidence Based Nursing, 12(4), 99. doi:10.1136/ ebn.12.4.99-b

ECRI Institute. (2019). Retrieved from https://guidelines.ecri.org/ Gobbo, S., Bergamin, M., Sieverdes, J. C., Ermolao, A., \& Zaccaria, M. (2014). Effects of exercise on dual-task ability and balance in older adults: A systematic review. Archives of Gerontology and Geriatrics, 58(2), 177-187. doi:10.1016/ j.archger.2013.10.001

Gray-Miceli, D., \& Quigley, P. A. (2013). Fall prevention. In M. Boltz, E. Capezuti, T. Fulmer, \& D. Zwicker (Eds.), Evidencebased geriatric nursing protocols for best practice (4th ed.). New York, NY: Springer Publishing Company. Retrieved from https://www.guidelinecentral.com/summaries/fall-prevention -in-evidence-based-geriatric-nursing-protocols-for-best -practice/\#section-society

Higgins, J. P. T., \& Green, S. (Eds.). (2011). Cochrane handbook for systematic reviews of interventions (Version 5.1.0). Retrieved from https://handbook-5-1.cochrane.org

Homan, J. M. (2010). Eyes on the prize: Reflections on the impact of the evolving digital ecology on the librarian as expert intermediary and knowledge coach, 1969-2009. Journal of the Medical Library Association, 98(1), 49-56. doi:10.3163/1536-5050.98.1.016

Keefer, J. M., \& Levin, R. F. (2013). Integration of critical thinking and EBP into "routine" practice. In R. F. Levin \& H. R. Feldman (Eds.), Teaching evidence-based practice in nursing (2nd ed., pp. 85-101). New York, NY: Springer Publishing Company.

Lefebvre, C., Manheimer, E., \& Glanville, J. (2011). Searching for studies. In J. P. T. Higgins \& S. Green (Eds.), Cochrane handbook for systematic reviews of interventions (Version 5.1.0, Chapter 6). Retrieved from https://handbook-5-1.cochrane.org

Levin, R. F. (2011). Edifices of evidence: The proliferation of pyramids. Research and Theory for Nursing Practice, 25(1), 8-10. doi:10.1891/1541-6577.25.1.8 
Levin, R. F., Singleton, J. K., \& Jacobs, S. K. (2008). Developing and evaluating clinical practice guidelines: A systematic approach. In E. Capezuti, D. Zwicker, M. D. Mezey, T. Fulmer, D. Gray-Miceli, \& M. Kluger (Eds.), Evidence-based geriatric nursing protocols for best practice (3rd ed., pp. 1-8). New York, NY: Springer Publishing Company.

Lucas, J. A., \& Fulmer, T. (2003). Evaluating clinical practice guidelines: A best practice. In M. D. Mezey, T. Fulmer, \& A. Ivo (Eds.), Geriatric nursing protocols for best practice (2nd ed., pp. 1-14). New York, NY: Springer Publishing Company.

McLellan, F. (2001). 1966 and all that-When is a literature search done? The Lancet, 358(9282), 646. doi:10.1016/ S0140-6736(01)05826-3

Melnyk, B. M., \& Fineout-Overholt, E. (2015). Evidence-based practice in nursing \& healthcare: A guide to best practice (3rd ed.). Philadelphia, PA: Lippincott Williams and Wilkins.

National Information Center on Health Services Research and Health Care Technology. (n.d.). Health economics information resources: A self-study course. Retrieved from https:// www.nlm.nih.gov/nichsr/edu/healthecon/01_he_12.html
Pravikoff, D. S., Tanner, A. B., \& Pierce, S. T. (2005). Readiness of U.S. nurses for evidence-based practice. American Journal of Nursing, 105(9), 40-51. doi:10.1097/00000446-2005 09000-00025

Slade, S. (2013). Falls (older adults): Preventative interventions. Retrieved from Joanna Briggs Institute EBP library database. https://joannabriggs.org/ebp

Smith, J., Forster, A., \& Young, J. (2006). Use of the "STRATIFY" falls risk assessment in patients recovering from acute stroke. Age and Ageing, 35(2), 138-143. doi:10.1093/ageing/afj027

Straus, S. E., Glasziou, P., Richardson, W. S., \& Haynes, R. B. (2010). Evidence-based medicine: How to practice and teach it (4th ed.). Edinburgh, UK: Churchill Livingstone.

Titler, M. (2010). Iowa model of evidence-based practice. In J. Rycroft-Malone \& T. Bucknall (Eds.), Models and frameworks for implementing evidence-based practice: Linking evidence to action. (pp. 137-146). Chichester, UK: Wiley-Blackwell.

Wentz, R. (2002). Visibility of research: FUTON bias. The Lancet, 360(9341), 1256. doi:10.1016/S0140-6736(02)11264-5 